\title{
The Reduced Phase Space of An Open String in The Background B-Field
}

\author{
M. Dehghani ${ }^{1}$, A. Shirzad ${ }^{2}$ \\ Department of Physics, Isfahan University of Technology \\ Isfahan, IRAN, \\ Institute for Studies in Theoretical Physics and Mathematics \\ P. O. Box: 5531, Tehran, 19395, IRAN.
}

\begin{abstract}
The problem of an open string in background $B$-field is discussed. Using the discretized model in details we show that the system is influenced by infinite number of second class constraints. We interpret the allowed Fourier modes as the coordinates of the reduced phase space. This enables us to compute the Dirac brackets more easily. We prove that the coordinates of the string are non-commutative at the boundaries. We argue that in order to find the Dirac bracket or commutator algebra of the physical variables, one should not expand the fields in terms of the solutions of the equations of motion. Instead, one should impose the set of constraints in suitable coordinates.
\end{abstract}

Keywords: Boundary conditions, Constraints, Dirac bracket, Reduced phase space, Non-commutativity.

${ }^{1}$ e-mail: mdehghani@ph.iut.ac.ir

${ }^{2}$ e-mail: shirzad@ipm.ir 


\section{Introduction}

The constrained systems were first introduced by Dirac [1] within the discussion about singular Lagrangians. In such systems, Euler-Lagrange equations of motion lead to some acceleration-free identities as consequences of the singularity of Hessian (second derivative of Lagrangian with respect to the velocities). At the Hamiltonian level, this leads to emergence of constraints, i.e. functions of phase space coordinates which should vanish on-shell.

We call that the first class constraints are responsible for gauge transformations; while second class constraints restrict the system to a smaller sub-manifold of the phase space in which a Poisson structure can be recognized. In a very simplified picture, the first class constraints may be visualized as some momenta, so that they are involutive and generate transformations in their conjugate coordinates (i.e. the gauged variables). On the other hand, the second class constraints may be visualized as conjugate pairs of coordinates and momenta whose Poisson brackets in the original phase space are nonzero. This seems to contradict the fact that they vanish on the surface of motion. Hence, it is necessary to introduce a new bracket, i.e. the Dirac bracket, such that the physical quantities (quantities defined on the constraint surface) have vanishing brackets with the constraints. A complete review on constrained systems can be found in [2]

The singularity of the Lagrangian is not necessarily the only origin of the constraints. One may find constraints in the Lagrangian formalism in any way that one can impose acceleration-free equations on the system; or in the Hamiltonian formalism, in any way that one can impose a primary constraint on the system. As far as we know, apart from artificial problems in which primary Hamiltonian constraints are imposed by hand, no serious model has been introduced in which constraints emerge naturally, but not as the result of the singularity of the system.

In the latest years of the last millennium, in another branch of research, a new phenomenon was discovered which has relationships with the constrained systems. It was found that for an open string coupled to a background $B$-field, the canonical quantization procedure fails at the end points $[3,4,5]$. It was also observed in Refs. [6, 7] based on the work of Ref. [8], that in the presence of mixed boundary conditions due to $B$-field, the propagators of coordinate fields possess singularities which can be interpreted as the non-commutativity of coordinate fields specially at the boundaries (branes). The origin of this non-commutativity is intensively discussed from then on $[9,10,11,12,13,14,15,16]$.

This observation led some authors $[4,5,9]$ to deduce that the Dirac mechanism of second class constraints has some role at the boundaries of the string, hence the idea of considering the boundary conditions as Dirac constraints was born $[4,5]$. Since the boundary conditions just put limitations on the solutions of equations of motion; if accepted as constraints, they should somehow be related to the second class systems.

In the next section we will review the main set up of the above model, following mainly the method of reference [9]. Assuming the boundary conditions as constraints, we use the 
total Hamiltonian to impose their consistency. As we will see, the Lagrange multipliers are determined at the first stage of consistency (to be zero), but at the same time the second level constraints emerge. Then third level constraints are derived by demanding the consistency of second level ones, and so forth. In this way the procedure of finding the constraints proceeds unlimitedly. This suggests a new category in comparison with the ordinary theory of constrained systems in which the constraint chains terminate after a limited number of steps either by arriving at an identity (for first class systems), or by determining the Lagrange multipliers (for second class systems) [17].

During recent years several different and even opposite approaches have been applied to the problem, though most of them have derived similar results. Some authors do not consider the consistency of the constraints completely. They calculate the Dirac brackets just by using primary or at most second level constraints [10, 18, 19]. The idea of infinite number of second class constraints at the boundaries, however, was accepted by many authors $[4,5,9]$, although fewer people note what happens to the Lagrange multipliers. Some authors have also tried to overcome the problem by considering discretized model [9] or by following the symplectic approach [11, 20, 21]. Such approaches, though verify that the system is constrained in some sense, do not give final justification about the constraint characteristics of the system.

Our first objective in this work is to find a distinctive understanding about the constraint structure of the model. We want to know in what sense we find infinite number of constraints. We think that the key to study this part of the problem is the discretized model. It is known that the primary constraints emerge as the continuum limit of the equations of motion of the end points in the discretized model [9, 10]. However investigating the consistency conditions to deduce the set of secondary constraints has not been studied in discretized model yet. In sections (3) and (4) we have done this. We will see that considering the continuum limit together with the physical condition of continuity gives the set of desired constraints. We think that in the light of this study of discrete model any doubt about the existence of infinite number of constraints disappears.

Next problem concerns the properties of the reduced phase space. Specially, one needs to know about the induced brackets on this space, which is the same as the Dirac brackets. With infinite number of constraints, the matrix of Poisson brackets of constraints is infinite dimensional. One needs to invert it to find the Dirac brackets. Some authors, which have accepted the existence of infinite number of constraints [5, 9], have tried to solve this problem directly. However, as we will explain in the following section, the mathematical manipulations given in different papers are not convincing yet. In fact, since people excepted to end up with non-commutativity in brane coordinates upon physical intuitions, most of the authors (except a few of them) have derived the famous result of Ref. [6] with more or less problematic mathematical methods.

In section (5) we will try to give a very simple approach to find the Dirac brackets on the basis of Fourier modes. We show that this powerful physical tool, (considered in some different sense in Ref. [22]) serves as the suitable coordinates for describing the reduced 
phase space. From this point of view, after imposing the constraints, the brackets of the remaining physical modes can be written automatically; and then the Dirac brackets of the original fields can be derived, using their expansion in terms of physical modes. Apart from convenience in calculations, this method helps one understand better the mechanism which leads to unusual brackets of coordinate fields at the boundaries.

In section(6) we give a comprehensive discussion about using the classical equations of motion of the fields in the process of quantization. We argue that in fact, the algebra of the observables is the essential entity in the quantization process rather than their dynamics. We show that just the dynamics of constraints is essential to be investigated before quantization. In this sense, we have derived the quantum properties of the reduced phase space, including the non-commutativity of brane coordinates, without using the solutions of the equations of motion.

\section{Problem setup}

Consider an open string with coordinate fields $X^{\mu}$ living in a target space specified by $\mu=0,1, \ldots, D$. Suppose that the string is coupled to a given antisymmetric field $\mathcal{F}_{\mu \nu}$ which for simplicity we assume no dynamics for it. The end points $\sigma=0, l$ of the string are constrained to move on a $p+1$ dimensional $D_{p}$-brane characterized by $X^{a}=0$ for $a=p+1, \ldots, D$. The $U(1)$ gauge fields, $A_{i} i=0,1, \ldots, p$ are also coupled to the string on the boundary $\left(D_{p}\right.$-brane). The action of the model can now be written as

$$
S=\frac{1}{4 \pi \alpha^{\prime}} \int_{\Sigma} d \sigma d \tau\left[G_{\mu \nu} \partial_{a} X^{\mu} \partial_{b} X^{\nu} g^{a b}+\epsilon^{a b} B_{\mu \nu} \partial_{a} X^{\mu} \partial_{b} X^{\nu}\right]+\frac{1}{2 \pi \alpha^{\prime}} \oint_{\partial \Sigma} d \tau A_{i} \partial_{\tau} X^{i}
$$

where $\partial \Sigma$ is the boundary of target space $\Sigma, g_{a b}$ is the metric of world-sheet and $\epsilon^{a b}$ is the antisymmetric tensor on the world-sheet. We considered for simplicity only the bosonic sector. Similar arguments can be applied to a superstring [13, 23]. The bulk and boundary fields can be combined to construct modified Born-Infeld field strength $\mathcal{F}=B-d A$. Let also both end points attach to the same brane. Assume $\alpha^{\prime}=\frac{1}{2 \pi}$ and the background metric to be flat: $G_{\mu \nu}=\eta_{\mu \nu}$. Suppose, moreover, that the field strength is constant everywhere, given by the constant antisymmetric matrix $B_{i j}$. Using these simplifications the action reads:

$$
S=\frac{1}{2} \int d \sigma d \tau\left[\partial_{a} X^{\mu} \partial_{b} X_{\mu} g^{a b}+B_{i j} \partial_{a} X^{i} \partial_{b} X^{j} \epsilon^{a b}\right]
$$

fixing the diffeomorphisms and scaling invariance, the above action takes the form

$$
S=\frac{1}{2} \int d \sigma d \tau\left[\dot{X}^{\mu} \dot{X}_{\mu}-X^{\prime \mu} X_{\mu}^{\prime}+2 B_{i j} \dot{X}^{i} X^{\prime j}\right]
$$

where "dot" and "prime" represent differentiating with respect to $\tau$ and $\sigma$ respectively. Besides the conformal symmetry, this action has a global symmetry under transforming the coordinate fields by a constant, i.e.

$$
X^{\mu}(\sigma, \tau) \rightarrow X^{\mu}(\sigma, \tau)+c^{\mu}
$$


Varying the action with respect to the fields $X^{\mu}$ gives the equations of motion

$$
\partial_{\tau}^{2} X^{\mu}(\sigma, \tau)-\partial_{\sigma}^{2} X^{\mu}(\sigma, \tau)=0 \quad \mu=0,1, \ldots, D
$$

together with the boundary conditions

$$
\begin{array}{ll}
\partial_{\sigma} X^{i}(\sigma, \tau)+B_{i j} \partial_{\tau} X^{j}(\sigma, \tau)=0 & i=0,1, \cdots, p \\
X^{a}=0 & a=p+1, \cdots, D
\end{array}
$$

at the end points $\sigma=0, l$. As is apparent the boundary conditions in the directions perpendicular to the $D_{p}$-brane are simply of Dirichlet type; while along the $D_{p}$-brane we have mixed boundary conditions. This is the only place where the effect of the $B$-field is experienced. Let concentrate in the following on this part of the problem and ignore the coordinate fields with Dirichlet boundary conditions. One simple way to realize this point is to assume that $p=D$. On the other hand, at the end of calculations one can turn off the $B$-field in as many direction as one desires to achieve results concerning the Neumann boundary conditions. The equations (2.6) do not contain accelerations, so it may be viewed as primary Lagrangian constraints. The canonical momentum fields and the Hamiltonian of the system read

$$
\begin{gathered}
\Pi_{i}(\sigma, \tau)=\partial_{\tau} X(\sigma, \tau)+B_{i j} \partial_{\sigma} X_{j}(\sigma, \tau) \\
H=\frac{1}{2} \int_{0}^{l}\left[\left(\Pi_{i}-B_{i j} \partial_{\sigma} X_{j}\right)^{2}+\left(\partial_{\sigma} X_{i}\right)^{2}\right] d \sigma .
\end{gathered}
$$

The primary constraints (2.6) in terms of the phase space variables are

$$
\Phi_{i}=\left.\Phi_{i}(\sigma)\right|_{\sigma=0}, \quad \bar{\Phi}_{i}=\left.\Phi_{i}(\sigma)\right|_{\sigma=l}
$$

with

$$
\Phi_{i}(\sigma, \tau)=M_{i j} \partial_{\sigma} X_{j}(\sigma, \tau)+B_{i j} \Pi_{j}(\sigma, \tau), \quad M=1-B^{2} .
$$

As for every constrained system, given the primary constraints (2.9) the total Hamiltonian

$$
H_{T}=H+\lambda_{i} \Phi_{i}^{1}+\bar{\lambda}_{i} \bar{\Phi}_{i}^{1}
$$

is responsible for the dynamics of the system. Hence, the consistency of the constraints gives

$$
\begin{aligned}
& \dot{\Phi}_{i}^{1}=\left\{\Phi_{i}^{1}, H_{T}\right\}=\left\{\Phi_{i}^{1}, H\right\}+\lambda_{j}\left\{\Phi_{i}^{1}, \Phi_{j}^{1}\right\}=0 \\
& \dot{\bar{\Phi}}_{i}^{1}=\left\{\bar{\Phi}_{i}^{1}, H_{T}\right\}=\left\{\bar{\Phi}_{i}^{1}, H\right\}+\bar{\lambda}_{j}\left\{\bar{\Phi}_{i}^{1}, \bar{\Phi}_{j}^{1}\right\}=0 .
\end{aligned}
$$

Using Eqs. (2.8-2.10) and the fundamental Poisson brackets

$$
\begin{aligned}
& \left\{X_{i}(\sigma, \tau), X_{j}\left(\sigma^{\prime}, \tau\right)\right\}=0 \\
& \left\{X_{i}(\sigma, \tau), \Pi_{j}\left(\sigma^{\prime}, \tau\right)\right\}=\delta_{i j} \delta\left(\sigma-\sigma^{\prime}\right) \\
& \left\{\Pi_{i}(\sigma, \tau), \Pi_{j}\left(\sigma^{\prime}, \tau\right)\right\}=0
\end{aligned}
$$


it is easy to see that

$$
\begin{gathered}
\Phi_{i}^{2} \equiv\left\{\Phi_{i}^{1}, H\right\}=\left.\partial_{\sigma} \Pi_{i}\right|_{\sigma=0} \\
\left\{\Phi_{i}^{1}, \Phi_{j}^{1}\right\}=-2(M B)_{i j} \int \delta(\sigma) \delta\left(\sigma^{\prime}\right) \partial_{\sigma} \delta\left(\sigma-\sigma^{\prime}\right) d \sigma d \sigma^{\prime} .
\end{gathered}
$$

Similar equations also arise for $\bar{\Phi}_{i}$ at the end point $\sigma=l$. Let see how can the consistency conditions (2.12) come true. Noticing (2.14) and (2.15), we find that the two Poisson brackets appearing on the right hand side of equations (2.12) are not of the same order. This fact is explained in more detail in [9] using the regularized form of the Dirac delta functions. Therefore the only way to satisfy the consistency conditions (2.12), is to assume that

$$
\begin{gathered}
\lambda_{j}=\bar{\lambda}_{j}=0, \\
\Phi_{i}^{2}=\bar{\Phi}_{i}^{2}=0 .
\end{gathered}
$$

The important result is that the secondary constraints emerge while the Lagrange multipliers are determined. One should continue the consistency process by demanding the time derivatives of $\Phi_{i}^{2}$ and $\bar{\Phi}_{i}^{2}$ to vanish. From (2.11) and (2.16) we have $H_{T}=H$ from now on, so

$$
\Phi_{i}^{3}=\dot{\Phi}_{i}^{2}=\left\{\Phi_{i}^{2}, H\right\}=\left.\partial_{\sigma}^{2} \Phi(\sigma, \tau)\right|_{\sigma=0}=0
$$

with similar expressions for $\bar{\Phi}_{i}^{3}$. In this way two infinite constraint chains appear as

$$
\begin{aligned}
\Phi_{i}^{n} & = \begin{cases}\left.\partial_{\sigma}^{n-1} \Phi_{i}\right|_{\sigma=0} & n=1,3, \cdots \\
\left.\partial_{\sigma}^{n-1} \Pi_{i}\right|_{\sigma=0} & n=2,4, \cdots\end{cases} \\
\bar{\Phi}_{i}^{n} & = \begin{cases}\left.\partial_{\sigma}^{n-1} \Phi_{i}\right|_{\sigma=l} & n=1,3, \cdots \\
\left.\partial_{\sigma}^{n-1} \Pi_{i}\right|_{\sigma=l} & n=2,4, \cdots\end{cases}
\end{aligned}
$$

Next the problem arises: what are the Dirac brackets of the fields due to the above infinite constraints. We recall that the Dirac bracket of two quantities $f$ and $g$ in phase space is defined as

$$
\{f, g\}_{D . B}=\{f, g\}+\left\{f, \chi_{i}\right\} C^{i j}\left\{\chi_{j}, g\right\}
$$

where $\chi_{i}$ are second class constraints and $C^{i j}$ is the inverse of

$$
C_{i j}=\left\{\chi_{i}, \chi_{j}\right\} .
$$

In the present problem $C_{i j}$ is infinite dimensional and it is difficult (or in fact impossible) to find its inverse. It is obvious that for all $m, n, i$ and $j$

$$
\left\{\Phi_{i}^{n}, \bar{\Phi}_{j}^{m}\right\}=0 .
$$

Therefore, the Dirac bracket (2.20) contains two separate parts, one due to inverse of $C_{i j}^{n m}=\left\{\Phi_{i}^{n}, \Phi_{j}^{m}\right\}$ and the other due to $\bar{C}_{i j}^{n m}$, defined similarly. So it is enough to do the calculations just for the set of $\Phi$ 's. Using the integral form of the constraints:

$$
\begin{aligned}
\Phi_{i}^{n} & =\int d \sigma \delta(\sigma) \partial_{\sigma}^{n} \Phi_{i}(\sigma, \tau) & n=0,2, \cdots \\
\Phi_{i}^{n} & =\int d \sigma \delta(\sigma) \partial_{\sigma}^{n} \Pi_{i}(\sigma, \tau) & n=1,3, \cdots
\end{aligned}
$$


it is straightforward to calculate

$$
C_{i j}^{n m}= \begin{cases}0 & n, m=1,3, \cdots \\ -2(M B)_{i j} \int \delta(\sigma) \delta\left(\sigma^{\prime}\right) \partial_{\sigma}^{m+1} \partial_{\sigma^{\prime}}^{n} \delta\left(\sigma-\sigma^{\prime}\right) d \sigma d \sigma^{\prime} & n, m=0,2, \cdots \\ M_{i j} \int \delta(\sigma) \delta\left(\sigma^{\prime}\right) \partial_{\sigma}^{m+1} \partial_{\sigma^{\prime}}^{n} \delta\left(\sigma-\sigma^{\prime}\right) d \sigma d \sigma^{\prime} & n=1,3, \cdots \quad m=0,2, \cdots\end{cases}
$$

This matrix possesses a finite dimensional part with $i, j$ indices and an infinite dimensional part with $m, n$ indices. The finite part can be inverted easily, while for infinite part there exist serious difficulties. In fact the most crucial point in studying the problem is here. Among so many authors that escaped the existence of infinite number of constraints, there are two main references, published almost simultaneously, that have tried to invert the matrix $C_{i j}^{n m}$ above, and calculate the Dirac brackets. First, the famous work of Ref. [5] in which the authors tried to write down the inverse of $C_{i j}^{n m}$ by using some undetermined functions $R_{n m}\left(\sigma^{\prime}, \sigma^{\prime \prime}\right)$ and $S_{n m}\left(\sigma^{\prime}, \sigma^{\prime \prime}\right)$, where it is claimed that these functions are omitted during calculating the Dirac brackets. However, we think that it is not allowed to do calculations for the midpoints $\sigma^{\prime}, \sigma^{\prime \prime}, \ldots$ of the string and thereafter consider them at the end points $\sigma=0, l$. In other words, the presence of $\delta(\sigma)$ and $\delta\left(\sigma^{\prime}\right)$ in the formula (2.24) is essential. In fact, it may happen that some expressions vanish for intermediate points of the string before going to the boundaries. ${ }^{3}$ In this way it is not clear that in what sense may the functions $R_{n m}\left(\sigma^{\prime}, \sigma^{\prime \prime}\right)$ and $S_{n m}\left(\sigma^{\prime}, \sigma^{\prime \prime}\right)$ be defined appropriately.

The next reference in this regard is Ref. [9], in which the authors have tried to regularize the delta functions in $(2.24)$ and then invert $C_{i j}^{n m}$. However, in practice this method does not seem to make it possible to find $C^{-1}$ directly. The authors have written the answer by considering some desired properties of Dirac brackets. In this way the main features of the answer is derived, but unfortunately it contains the regularization parameter of delta functions, which does not sound plausible. The reality is that, apart from some exceptional references[19, 16], most authors have tried to find, in different ways, the original results which imply the non-commutativity of coordinate fields $X^{\mu}$ at the boundaries (see Eqs. (5.23) in the following). We will show in section (5) that applying the familiar approach of mode expansion in the context of constrained systems gives reliable results.

Before that we prefer to spend two sections to establish the existence of infinite number of constraints by studying the fundamental discrete model corresponding to the continuum model.

\footnotetext{
${ }^{3}$ This precise point made the author of [19] to deduce that the first level constraints commute, and finally that the coordinate field are commutative at the boundaries after quantization. Being more accurate $\left[\Phi(\sigma, \tau), \Phi\left(\sigma^{\prime}, \tau\right)\right]=0$ for arbitrary $\sigma, \sigma^{\prime}$ due to antisymmetry of $B$, while using (2.23) shows that at boundary $\sigma=0,[\Phi(0, \tau), \Phi(0, \tau)] \neq 0$ as can be seen from $(2.24)$.
} 


\section{Discretization}

The discretized Lagrangian corresponding to the model given in (2.3) can be written as:

$$
L=\frac{1}{2} \epsilon \sum_{n=0}^{N}\left(\dot{X}_{n}\right)^{2}-\frac{1}{2} \epsilon \sum_{n=0}^{N-1}\left(\frac{X_{n+1}-X_{n}}{\epsilon}\right)^{2}+\sum_{n=0}^{N-1} \dot{X}_{n} B\left(X_{n+1}-X_{n}\right)
$$

For the sake of simplicity in notation we have dropped the $i, j$ indices on $B$-field. So $B$ should be considered as a matrix and $X_{n}$ as a column vector in the above equation as well as in the following. All associated quantities to $X_{n}$ carry the same hidden index of a column vector. The continuum limit is achieved by the following replacements:

$$
\begin{aligned}
& N \rightarrow \infty \\
& \epsilon \rightarrow 0 \\
& N \epsilon \rightarrow l \\
& n \epsilon \rightarrow \sigma \\
& X_{n} \rightarrow X(\sigma, \tau) \\
& \frac{X_{n+1}-X_{n}}{\epsilon} \rightarrow \partial_{\sigma} X(\sigma, \tau) .
\end{aligned}
$$

Here we have ascribed the right difference (divided by $\epsilon$ ) to spatial derivative. It is also possible to do the same with the left difference. In the continuum limit the physical quantities in the neighboring points are the same.

The Euler-Lagrange equations of motion for intermediate points are:

$$
\ddot{X}_{n}-\frac{\Delta_{n}}{\epsilon^{2}}+\epsilon B \frac{\dot{\Delta}_{n}}{\epsilon^{2}}=0
$$

where

$$
\Delta_{n} \equiv X_{n+1}-2 X_{n}+X_{n-1}
$$

The last term in (3.3) is of order $\epsilon$ and vanishes in the continuum limit (3.2) giving the wave equation (2.5) which implies that the $B$-field has no effect in the intermediate points and appears only in the equations of motion of the boundary points, as follows

$$
\begin{aligned}
& \epsilon \ddot{X}_{0}-\frac{X_{1}-X_{0}}{\epsilon}-B\left(2 \dot{X}_{0}-\dot{X}_{1}\right)=0 \\
& \epsilon \ddot{X}_{N}+\frac{X_{N}-X_{N-1}}{\epsilon}+B \dot{X}_{N-1}=0
\end{aligned}
$$

In the continuum limit the terms proportional to $\epsilon$ in Eqs. (3.5) vanish, while $\dot{X}_{1}$ and $\dot{X}_{N-1}$ can be replaced by $\dot{X}_{0}$ and $\dot{X}_{N}$ respectively, to obtain the following acceleration-free equations

$$
\partial_{\sigma} X(\sigma, \tau)+\left.B \dot{X}(\sigma, \tau)\right|_{\sigma=0, l}=0 .
$$

Eqs. (3.6) are the primary Lagrangian constraints. In the Hamiltonian formalism the momenta conjugate to coordinates $X_{n}$ are

$$
\begin{aligned}
& p_{n}=\epsilon \dot{X}_{n}+B\left(X_{n+1}-X_{n}\right) \quad n=0,1, \cdots, N-1 \\
& p_{N}=\epsilon \dot{X}_{n} .
\end{aligned}
$$


The canonical Hamiltonian reads:

$$
H=\frac{1}{2 \epsilon} \sum_{n=0}^{N-1}\left[\left(p_{n}-B\left(X_{n+1}-X_{n}\right)\right)^{2}+\left(X_{n+1}-X_{n}\right)^{2}\right]+\frac{1}{2 \epsilon} p_{N}^{2} .
$$

To achieve the continuum limit one should complete the list given in (3.2) as follows:

$$
\begin{aligned}
p_{n} & \rightarrow 0 \quad n=0,1, \cdots, N \\
\frac{p_{n}}{\epsilon} & \rightarrow \Pi(\sigma, \tau)=\dot{X}(\sigma, \tau)+B \partial_{\sigma} X(\sigma, \tau) \quad n=0,1, \cdots, N-1 \\
\frac{H}{\epsilon} & \rightarrow \mathcal{H}\left(\Pi(\sigma, \tau), X(\sigma, \tau), \partial_{\sigma} X(\sigma, \tau)\right)
\end{aligned}
$$

where in the second line Eq.(3.7) is used and $\Pi(\sigma, \tau)$ and $\mathcal{H}$ are the momentum field and Hamiltonian density, respectively. The canonical equations of motion for the intermediate points are as follows:

$$
\begin{gathered}
\dot{p}_{n}=\frac{1}{\epsilon}\left[B\left(p_{n}-p_{n-1}\right)+M \Delta_{n}\right] \quad n=1,2, \cdots, N-1 \\
\dot{X}_{n}=\frac{1}{\epsilon}\left[p_{n}-B\left(X_{n+1}-X_{n}\right)\right] \quad 0 \leq n<N .
\end{gathered}
$$

For the end points we have

$$
\begin{aligned}
& \dot{p}_{0}=\frac{1}{\epsilon}\left[B p_{0}+M\left(X_{1}-X_{0}\right)\right], \\
& \dot{p}_{N}=-\frac{1}{\epsilon}\left[B p_{N-1}+M\left(X_{N}-X_{N-1}\right)\right] .
\end{aligned}
$$

The right hand side of the above equations are finite in the continuum limit, while the left hand side vanishes (see Eqs.( 3.9)). So Eqs. (3.12) can be viewed as the following primary Hamiltonian constraints

$$
\Phi^{1}=M \partial_{\sigma} X(\sigma, \tau)+\left.B \Pi(\sigma, \tau)\right|_{\sigma=0, l}
$$

It should be noticed that $\left(p_{N}-p_{N-1}\right)$ is of order $\epsilon^{2}$, so in the continuum limit $p_{N-1} / \epsilon$ can also represent the the end point momentum $\Pi(\sigma=l, \tau)$ as well as $p_{N} / \epsilon$. For the same reason $\left(X_{N}-X_{N-1}\right) / \epsilon$ can be interpreted as $\left.\partial_{\sigma} X(\sigma, \tau)\right|_{\sigma=l}$ despite our previous convention of attribution of right difference to the spatial derivative at a given point. These constraints can also be derived from Lagrangian constraints (3.5) upon inserting $\dot{X}(\sigma, \tau)$ from (2.7). The constraints at the end points are completely similar to each other. So without losing any point, we can concentrate only on the boundary $\sigma=0$. The same arguments can be established for the other boundary $\sigma=l$.

\section{Consistency condition for the constraints}

In the previous section we showed that the equations of motion for the end points of the string can be treated as primary constraints. As in any constrained system one should 
investigate the consistency of the constraints. In discrete model this means that one should differentiate the equations producing the constraints, i.e. Eqs. (3.12) with respect to time to give

$$
\frac{1}{\epsilon}\left[B \dot{p}_{0}+M\left(\dot{X}_{1}-\dot{X}_{0}\right)\right]=O(\epsilon)
$$

Using Eq. (3.11) to insert $\dot{X}_{0}$ and $\dot{X}_{1}$ into (4.1) gives

$$
\frac{1}{\epsilon} B \dot{p}_{0}+\frac{1}{\epsilon^{2}} M\left(-B \Delta_{1}+p_{1}-p_{0}\right)=O(\epsilon) .
$$

Inserting $M \Delta_{1}$ from $\mathrm{Eq}(3.10)$ in $\mathrm{Eq}(4.2)$ results in

$$
\frac{1}{\epsilon} B\left(\dot{p}_{0}-\dot{p}_{1}\right)+\frac{1}{\epsilon^{2}}\left(p_{1}-p_{0}\right)=O(\epsilon)
$$

The first term is of order $\epsilon$ while the second term is the discrete version of $\left.\partial_{\sigma} \Pi(\sigma, \tau)\right|_{\sigma=0}$. Therefore in the limit $\epsilon \rightarrow 0$ the second level constraint emerges as

$$
\Phi^{2}=\left.\partial_{\sigma} \Pi(\sigma, \tau)\right|_{\sigma=0}=0
$$

One should proceed the consistency condition for the new constraint $\Phi^{2}$. Due to some technical difficulties if we wish to do this in the discrete model it is not a good idea to differentiate directly the discrete version $\frac{p_{1}-p_{0}}{\epsilon^{2}}$ of the constraint $\Phi^{2}$. Instead, it is better to represent $\Phi^{2}$ with $\frac{p_{2}-p_{1}}{\epsilon^{2}}$. In fact we can transfer the condition $\left.\partial_{\sigma} \Pi(\sigma, \tau)\right|_{\sigma=0}=0$ to the right by infinitesimal distance $\epsilon$. This is reasonable since in the continuum limit every local condition on the fields should be valid in a small neighborhood of a point, not just strictly at the given point. This is in fact the "continuity hypothesis". In other words, it is not plausible to go to continuum limit just by taking the limits given in equations (3.2) and (3.9). It is also needed to impose the continuity hypothesis on the physical quantities, which implies that the difference of discrete values of fields in the neighboring points can be at most of order $\epsilon$. Therefore, differentiating $\Phi^{2}=\frac{p_{2}-p_{1}}{\epsilon^{2}}+O(\epsilon)$ gives

$$
\Phi^{3}=\frac{1}{\epsilon^{2}}\left(\dot{p}_{2}-\dot{p}_{1}\right)+O(\epsilon)
$$

It is worth noting that differentiation with respect to time does not change the order of a quantity. The reason is that time derivative of a quantity is achieved by the Poisson bracket of that quantity with the Hamiltonian which is of order $\epsilon$; but in computing the Poisson bracket, one differentiates with respect to canonical momenta $p_{n}$, which are also of order $\epsilon$. So the net result is of the same order. We insert $\dot{p}_{1}$ and $\dot{p}_{2}$ from Eq. (3.10) into Eq. (4.5), to get

$$
\Phi^{3}=\frac{1}{\epsilon^{3}}\left[B\left(p_{2}-2 p_{1}+p_{0}\right)+M\left(X_{3}-3 X_{2}+3 X_{1}-X_{0}\right)\right]+O(\epsilon)
$$

Going to the continuum limit we have

$$
\Phi^{3}=\left.\left[B \partial_{\sigma}^{2} \Pi(\sigma, \tau)+M \partial_{\sigma}^{3} X(\sigma, \tau)\right]\right|_{\sigma=0}=\partial_{\sigma}^{2} \Phi^{1}
$$


where we have used (3.13).

To investigate the consistency of $\Phi^{3}$, the strategy is the same as before: we should differentiate $\Phi^{3}$ with respect to time, but we need to transfer the terms one step to the right. Then using the equations of motion (3.10), (3.11) and the continuity hypothesis, and going back to the continuum limit, one gets the next constraint as

$$
\Phi^{4}=\left.\partial_{\sigma}^{3} \Pi(\sigma, \tau)\right|_{\sigma=0}
$$

It is reasonable that this procedure will produce at the boundaries the infinite set of constraints

$$
\Phi^{1}, \quad \partial_{\sigma} \Pi, \quad \partial_{\sigma}^{2} \Phi^{1}, \quad \partial_{\sigma}^{3} \Pi, \cdots
$$

which is the same as (2.18) and (2.19). One may wonder about the validity of constraints not only at the end points, but also in an infinite number of their adjacent points. The answer is that a real continues system consists of uncountably infinite points, while in the discretized model one imposes the constraints on a countable infinite number of points in the vicinity of the boundaries. So physically speaking, nothing bad has happened. In other words, suppose we extend the validity of the constraint $\left.\partial_{\sigma} X(\sigma, \tau)\right|_{\sigma=0}=0$ (in the case of a simple string with free end points) to a large countable number of the adjacent points of the boundary. Even when the number of points goes to infinity, it will still remain in an infinitesimal neighborhood of the boundary. In other words, it will never extend in the continuum limit to a finite distance from the boundary.

\section{$5 \quad$ Reduced phase space}

In this section we try to find out the most suitable basis to describe the physical (reduced) phase space. Whenever second class constraints exist, one should first impose the constraints to eliminate the redundant variables and reach the reduced phase space. One should then try to find the most suitable bracket on the reduced phase space. It is clear that the ordinary canonical quantization procedure (i.e. converting the Poisson brackets to commutators) is not consistent in the original phase space, since quantum operators corresponding to constraints have non-vanishing commutators which contradicts the necessity that they should vanish either strongly or on the physical states. However, a consistent quantization procedure can be done in the reduced phase space. This is achieved by converting the induced brackets on the reduced phase space to commutators.

Fortunately the famous Darboux theorem ensures us that a unique and well defined bracket, which is the same as the Dirac bracket, exists on this space [24]. In fact, the Poisson bracket in the original phase space induces the Dirac bracket on the reduced phase space $[1,2]$. In other words, for any two functions $f(q, p)$ and $g(q, p)$ one can write

$$
\{f(q, p), g(q, p)\}_{D . B}=\left\{\left.f(q, p)\right|_{\Phi=0},\left.g(q, p)\right|_{\Phi=0}\right\}
$$

where $\left.f(q, p)\right|_{\Phi=0}$ means evaluation of $f(q, p)$ on the constraint surface described by the equations $\Phi=0$. 
In the general case, the second class constraints may be some complicated functions of the coordinates. If so, the constrained and physical degree of freedom are mixed with each other and it is not generally an easy task to separate them. Sometimes it is almost impossible to compute the Dirac brackets directly from the definition (2.20), as is the case for our current model (string in the background B-field). Moreover, it may happen that the resulted quantum algebra is difficult to handle, specially in order to find the corresponding representations.

Now consider an idealized model in which the second class constraints are given by $q_{k+1}, \cdots q_{N}, p_{k+1}, \cdots p_{N}$ where the coordinates $\left(q_{1} \cdots q_{N}, p_{1} \cdots p_{N}\right)$ describe the original phase space in which the Poisson brackets are defined as

$$
\{f, g\}=\sum_{i=1}^{N}\left(\frac{\partial f}{\partial q_{i}} \frac{\partial g}{\partial p_{i}}-\frac{\partial f}{\partial p_{i}} \frac{\partial g}{\partial q_{i}}\right)
$$

It is clear that the reduced phase space with coordinates $\left(q_{1} \cdots q_{k}, p_{1} \cdots p_{k}\right)$ acquires a natural bracket in which summations run from 1 to $k$, i.e.

$$
\{f, g\}_{D . B .}=\sum_{i=1}^{k}\left(\frac{\partial f}{\partial q_{i}} \frac{\partial g}{\partial p_{i}}-\frac{\partial f}{\partial p_{i}} \frac{\partial g}{\partial q_{i}}\right) .
$$

Such a truncated Poisson bracket is a realization of the instruction (5.1) and can be checked that is equal to the Dirac bracket of (2.20). In fact, after imposing the constraints on this system, the Dirac brackets of the remaining variables are the same as their Poisson brackets. Hence, quantization of the system may be achieved in the most simple way, such that the familiar algebra of $x-p$ variables and the corresponding representations are still valid.

Due to extreme simplicity of the above system, it is much more convenient to change the coordinates of a theory with second class constraints to a basis in which the constraints constitute a set of conjugate pairs. We call such a coordinate system as normal coordinates. In most physical theories the Fourier modes are normal coordinates. Traditionally people are used to impose assumed commutation relations among the Fourier coefficients in order to quantize a field. However, the important point, which is not clearly stated in the literature, is that the Fourier modes are the normal coordinates describing the reduced phase space. In other words, they carry the "net physics of the theory", hence they are independent variables which are suitable for quantization.

\subsection{Free bosonic string}

For example consider a free bosonic string with Neumann boundary conditions [9]. In this simple case we are given the conjugate fields $X(\sigma, \tau)$ and $\Pi(\sigma, \tau)$ with the following Poisson bracket

$$
\left\{X(\sigma, \tau), \Pi\left(\sigma^{\prime}, \tau\right)\right\}=\delta\left(\sigma-\sigma^{\prime}\right)
$$


Suppose the fields are real. The most general form of their Fourier expansion can be written as:

$$
\begin{aligned}
& X(\sigma, \tau)=\frac{1}{\sqrt{2 \pi}} \int_{-\infty}^{\infty} d k\left[a_{k}(\tau) \cos k \sigma+b_{k}(\tau) \sin k \sigma\right] \\
& \Pi(\sigma, \tau)=\frac{1}{\sqrt{2 \pi}} \int_{-\infty}^{\infty} d k\left[c_{k}(\tau) \cos k \sigma+d_{k}(\tau) \sin k \sigma\right] .
\end{aligned}
$$

It is obvious that $a_{k}(\tau)$ and $c_{k}(\tau)$ should be even functions of $k$ while $b_{k}(\tau)$ and $d_{k}(\tau)$ should be odd. Using (5.4) it is easy to see that $\left(a_{k}, c_{k}\right)$ and $\left(b_{k}, d_{k}\right)$ are conjugate pairs in the space of new variables, i.e.

$$
\left\{a_{k}(\tau), c_{k^{\prime}}(\tau)\right\}=\left\{b_{k}(\tau), d_{k^{\prime}}(\tau)\right\}=\delta\left(k-k^{\prime}\right)
$$

and all other Poisson brackets vanish. The second class constraints of the system are:

$$
\begin{aligned}
& \left.\partial_{\sigma}^{2 n+1} X(\sigma, \tau)\right|_{\sigma=0, l}=0 \\
& \left.\partial_{\sigma}^{2 n+1} \Pi(\sigma, \tau)\right|_{\sigma=0, l}=0
\end{aligned}
$$

which can be derived similar to section (2) or by imposing $B=0$ on its results. The constraints at $\sigma=0$, give

$$
\begin{aligned}
& \int_{-\infty}^{\infty} d k(-1)^{n} k^{2 n+1} b_{k}=0 \\
& \int_{-\infty}^{\infty} d k(-1)^{n} k^{2 n+1} d_{k}=0
\end{aligned}
$$

Since $b_{k}$ and $d_{k}$ are odd, Eqs. (5.8) can be satisfied for all $n$, iff $b_{k}=d_{k}=0$, hence Eqs. (5.5) change to

$$
\begin{aligned}
& X(\sigma, \tau)=\frac{1}{\sqrt{2 \pi}} \int_{-\infty}^{\infty} d k a_{k}(\tau) \cos k \sigma \\
& \Pi(\sigma, \tau)=\frac{1}{\sqrt{2 \pi}} \int_{-\infty}^{\infty} d k c_{k}(\tau) \cos k \sigma .
\end{aligned}
$$

This means that in the basis of Fourier modes, $b_{k}$ and $d_{k}$ are constrained variables and the reduced phase space is simply achieved by omitting them. This is the advantage of using the Fourier modes as normal coordinates. If we were insisting on working in the original basis $X(\sigma, \tau)$ and $\Pi(\sigma, \tau)$, we would encounter difficulties explained at the end of section (2). Now let us consider the constraints on the end point $\sigma=l$. They give

$$
\begin{aligned}
& \int_{-\infty}^{\infty} d k(-1)^{n} k^{2 n+1} a_{k} \sin k l=0 \\
& \int_{-\infty}^{\infty} d k(-1)^{n} k^{2 n+1} c_{k} \sin k l=0
\end{aligned}
$$

Since $a_{k}$ and $c_{k}$ are even, the integrands in Eqs. (5.10) are even with respect to $k$. So the only way to impose the constraints is:

$$
a_{k}=c_{k}=0 \quad \text { for } \quad k l \neq 0, \pi, 2 \pi, \ldots
$$

Once again we see the miracle of working with Fourier modes. In this basis a large class of the coordinates $a_{k}$ and $c_{k}$ are omitted due to the constraints, just remaining with those 
with discrete values for $k$ as $k=n \pi / l$ for integer $n$. Finally the original field variables can be expanded in terms of infinite countable Fourier modes $a_{n}$ and $c_{n}$ as canonical coordinates of the reduced phase space as:

$$
\begin{aligned}
& X(\sigma, \tau)=\frac{1}{\sqrt{l}} a_{0}(\tau)+\sqrt{\frac{2}{l}} \sum_{n=1}^{\infty} a_{n}(\tau) \cos \frac{n \pi \sigma}{l} \\
& \Pi(\sigma, \tau)=\frac{1}{\sqrt{l}} c_{0}(\tau)+\sqrt{\frac{2}{l}} \sum_{n=1}^{\infty} c_{n}(\tau) \cos \frac{n \pi \sigma}{l}
\end{aligned}
$$

It is easy to see that the Fourier modes $a_{m}$ and $c_{n}$ obey the canonical brackets:

$$
\left\{a_{m}, a_{n}\right\}=\left\{c_{m}, c_{n}\right\}=0, \quad\left\{a_{m}, c_{n}\right\}=\delta_{m n}
$$

Using expansion (5.12) and brackets (5.13), one can compute the Dirac brackets of any two physical functions of the original variables $X(\sigma, \tau)$ and $\Pi(\sigma, \tau)$. This means that we have followed the prescription given in Eq. (5.5) to find the Dirac brackets. As is well known [1], the second class constraints should strongly vanish before quantization. This fact can be stated in terms of the Fourier modes more clearly. The constrained modes $\left(b_{k}, d_{k}\right)$ for all $k$ and $\left(a_{k}, c_{k}\right)$ for $k \neq n \pi / l$ should vanish before quantization. Then one can quantize the theory by assuming canonical commutation relations among $\left(\hat{a}_{m}, \hat{c}_{m}\right)$ in the expansion (5.12) as

$$
\left[\hat{a}_{m}, \hat{c}_{n}\right]=i \hbar \delta_{m n}
$$

\subsection{Open string in background $B$-field}

Let us now consider the string in the background $B$-field. We expand the main fields $X(\sigma, \tau)$ and $\Pi(\sigma, \tau)$ as done in (5.5) with $\left(a_{k}, c_{k}\right)$ and $\left(b_{k}, d_{k}\right)$ as conjugate pairs. The constraints $(2.18)$ and $(2.19)$ can be rewritten as

$$
\begin{aligned}
& \left.\partial_{\sigma}^{2 n} \Phi(\sigma, \tau)\right|_{\sigma=0, l}=0 \\
& \left.\partial_{\sigma}^{2 n+1} \Pi(\sigma, \tau)\right|_{\sigma=0, l}=0
\end{aligned} \quad n=0,1,2, \cdots
$$

Using the constraints (5.15) at $\sigma=0$ read

$$
\begin{aligned}
& \int_{-\infty}^{\infty} d k(-1)^{n} k^{2 n}\left(k M b_{k}+B c_{k}\right)=0 \\
& \int_{-\infty}^{\infty} d k(-1)^{n} k^{2 n+1}\left(d_{k}\right)=0 .
\end{aligned}
$$

Remembering that $\left(b_{k}, d_{k}\right)$ are odd and $c_{k}$ is even with respect to $k$, the constraints $(5.16)$ are satisfied for all $n$ and $k \neq 0$ iff

$$
\begin{aligned}
& b_{k}=-\frac{1}{k} M^{-1} B c_{k} \\
& d_{k}=0 .
\end{aligned}
$$


Now imposing the constraints (5.15) on the end point $\sigma=l$ gives

$$
\begin{aligned}
& \int_{-\infty}^{\infty} d k(-1)^{n} k^{2 n}\left(-k M a_{k}\right) \sin k l=0 \\
& \int_{-\infty}^{\infty} d k(-1)^{n} k^{2 n+1}\left(c_{k}\right) \sin k l=0 .
\end{aligned}
$$

These equations show that $\left(a_{k}, c_{k}\right)$ and consequently $b_{k}$ should vanish for $k \neq n \pi / l(n$ integer). To this end, some care is needed for the zero mode $(k=0)$. In the limit $k \rightarrow 0$, using (5.17) we have

$$
\lim _{k \rightarrow 0} b_{k} \sin k \sigma=\lim _{k \rightarrow 0}\left(-\frac{1}{k} M^{-1} B c_{k} \sin k \sigma\right)=-M^{-1} B c_{0} \sigma .
$$

Therefore the linear term $\left(-M^{-1} B c_{0} \sigma\right)$, coming from the zero mode of the sine terms should be present in the expansion of $X(\sigma, \tau)$. Similarly to $(5.12)$ the term $\frac{1}{\sqrt{l}} a_{0}$ is also necessary as the zero mode of cosine terms. However according to the global symmetry given in (2.4), we are allowed to add any constant term to the expansion of $X(\sigma, \tau)$. This term should not disturb the validity of constraints (5.15) and should vanish in the limit $B \rightarrow 0$. We fix this arbitrariness by adding the constant term as $M^{-1} B c_{0} l / 2$. As we will see later, $c_{0}$ is constant according to the equations of motion and moreover, this choice guarantees that the coordinates of the center of mass of the string are commutative.

Putting all these results together, the most general form of the fields satisfying the constraints can be written as

$$
\begin{aligned}
& X(\sigma, \tau)=\frac{1}{\sqrt{l}}\left(a_{0}-M^{-1} B c_{0}\left(\sigma-\frac{l}{2}\right)\right)+\sqrt{\frac{2}{l}} \sum_{n=1}^{\infty}\left(a_{n} \cos \frac{n \pi \sigma}{l}-\frac{l}{n \pi} M^{-1} B c_{n} \sin \frac{n \pi \sigma}{l}\right), \\
& \Pi(\sigma, \tau)=\frac{1}{\sqrt{l}} c_{0}+\sqrt{\frac{2}{l}} \sum_{n=1}^{\infty} c_{n} \cos \frac{n \pi \sigma}{l} .
\end{aligned}
$$

These relations show that in the case of mixed boundary conditions again $a_{n}$ and $c_{n}$ are suitable canonical coordinates of the reduced phase space. Note that $\left(a_{n}, c_{n}\right)$, as canonical coordinates, still obey the canonical brackets (5.13). Hence, from the general prescription given in (5.1) it is easy to calculate the Dirac brackets of the fields, just by using their expressions in terms of normal coordinates $a_{n}$ and $c_{n}$. As can be seen from (5.20) the $B$-field appears only in the expansion of coordinate fields $X_{i}(\sigma, \tau)$, while the momentum fields $\Pi_{i}(\sigma, \tau)$ are unchanged. Therefore, the Dirac brackets $\left\{X_{i}(\sigma, \tau), \Pi_{j}\left(\sigma^{\prime}, \tau\right)\right\}_{D . B}$ and $\left\{\Pi_{i}(\sigma, \tau), \Pi_{j}\left(\sigma^{\prime}, \tau\right)\right\}_{D . B}$ are the same as the corresponding Poisson brackets given in Eqs. (2.13). However, for the Dirac brackets of coordinate fields, from (5.13) and (5.20), one finds

$$
\left\{X_{i}(\sigma, \tau), X_{j}\left(\sigma^{\prime}, \tau\right)\right\}_{D . B}=\left(M^{-1} B\right)_{i j}\left[\frac{\sigma+\sigma^{\prime}}{l}-1+\frac{2}{\pi} \sum_{n=1}^{\infty} \frac{1}{n} \sin \frac{n \pi}{l}\left(\sigma+\sigma^{\prime}\right)\right] .
$$

The summation over the sines is the Fourier expansion of saw waves as follows

$$
\sum_{n=1}^{\infty} \frac{1}{n} \sin n \theta= \begin{cases}-\frac{1}{2}(\pi+\theta) & -\pi \leq \theta<0 \\ \frac{1}{2}(\pi-\theta) & 0<\theta \leq \pi\end{cases}
$$


This function is discontinuous at $\theta=0,2 \pi, \ldots$. Supposing its values at these points to be the average of right and left limits, i. e. zero, we can write the final result as:

$$
\begin{aligned}
& \left\{X_{i}(\sigma, \tau), X_{j}\left(\sigma^{\prime}, \tau\right)\right\}_{D . B}=0 \quad \sigma, \sigma^{\prime} \neq 0 \\
& \left\{X_{i}(0, \tau), X_{j}(0, \tau)\right\}_{D . B}=-2\left(M^{-1} B\right)_{i j}, \\
& \left\{X_{i}(l, \tau), X_{j}(l, \tau)\right\}_{D . B}=2\left(M^{-1} B\right)_{i j} .
\end{aligned}
$$

As we see, after quantization the coordinate fields are noncommutative at the end-points of the string, in agreement with the well-known results given in the literature $[4,5,9]$. If we had not added the constant term $M^{-1} B c_{0} l / 2$ to the expansion (5.20) the above result would have differed from (5.23) just by a constant term throughout the string, as well as at the end-points. In other words the non-commutativity at the end-points $\sigma=0$ and $\sigma=l$ have opposite signs since we have imposed the condition that the center of mass coordinates are commutative.

Our emphasize in our derivation of the important result (5.23) is that we have not used the expansions of fields in terms of the solutions of the equations of motion. In fact, we have not considered the time dependence of the physical modes $a_{n}(\tau)$ and $c_{n}(\tau)$ which should be determined by means of the special form of the Lagrangian or Hamiltonian. This feature of our approach will be explained more in the next section.

\section{Quantization and equations of motion}

The traditional canonical quantization procedure is as follows: one considers the general solution of the classical equations of motion, then imposes the boundary conditions to decrease the number of possible modes ${ }^{4}$, and finally assumes suitable commutation relations amongst the physical modes to quantize the theory. One may ask: "is it really necessary, or even allowed, to use classical equations of motion in the process of quantization?".

Let us first study the problem in an ordinary (unconstrained) system. The important point is that the special form of the Hamiltonian (or Lagrangian) is not the essential point that determines the algebra of physical observables and consequently the structure of the space of physical states of the theory. On the other hand, the role of the Hamiltonian is just determining the dynamics of the system. Given the initial state of a system, the Hamiltonian is the main tool which gives the time evolution of the state of the system. However, people usually try to construct the basis of the space of the physical states in such a way that the Hamiltonian operator is diagonal; since this provides consequent convenience to follow the dynamics of the system.

For example in one dimension whatever the Hamiltonian is, one can use the algebra of the $x-p$ operators acting on the space with $\mid x^{\prime}>$ 's or $\mid p^{\prime}>$ 's as the basis, as well as the equivalent algebra of $a-a^{\dagger}$ operators and the corresponding basis in which the operator

\footnotetext{
${ }^{4}$ The classical equations of motion are usually linear differential equations; so one can expand their solutions using a complete set of modes
} 
$a^{\dagger} a$ is diagonal. However, it is well-known that for a free particle the former algebra is more suitable while for the harmonic oscillator the latter is more appropriate to study the dynamics of the system.

To this end, we want to emphasize that conceptually it is not needed to treat the quantum fields as the solutions of the classical equations of motion. However, the reader may have encountered several books or papers where the authors write down the fields as an expansion in terms of solutions of the classical equations of motion (for example plane waves with definite $\omega-k$ relations) and then quantize the theory by imposing assumed canonical algebra among the coefficients of the expansion. A careful notice leads to the observation that most of the time, the explicit time dependence of the terms in the expansion are not used during the subsequent analysis. For example in harmonic oscillator problem, it is just the quantum algebra $\left[a, a^{\dagger}\right]=1$, coming from the classical algebra $\{x, p\}_{P . B .}=1$ upon quantization, which determines the basis of physical states as $|n>| n+1>,, \cdots{ }^{5}$ Then the explicit form of the Hamiltonian may be used to determine the time dependence of $a(t)$ and $a^{\dagger}(t)$ in Heisenberg picture as $a(t)=a(0) \exp (-i \omega t)$ and $a^{\dagger}(t)=a^{\dagger}(0) \exp (i \omega t)$, which is not essential in determining the quantum properties of the observables as well as the space of physical states.

Our experience shows that in quantum field theory the Fourier expansion of the fields come out, most of the time, to be useful in the process of quantization. As discussed in the case of the models considered in this paper, this is just a change of variables in the phase space from $X(\sigma, \tau)$ and $\Pi(\sigma, \tau)$ say, to $a_{k}(\tau), b_{k}(\tau), \cdots$ etc. Then, regardless of the dynamics of the system, the Poisson brackets of the original variables determine those of the new ones. There are two main advantages in this change of variables. First, the Hamiltonian may be diagonal or have a simpler form in the new basis. Second, the constraints as well as boundary conditions (which are also considered as constraints in our approach) may be applied in a simpler way in the framework of the new variables. Therefore, the Hamiltonian has some partial role in quantization since the dynamics of constraints, (i.e. the consistency of the constraints) should be investigated classically before quantization. It is not possible to construct a quantum algebra among the variables, unless the Hamiltonian has vanishing brackets with the constraints on the physical space (reduced phase space). ${ }^{6}$

In other words, although the full dynamics of the physical variables is not essential for quantizing the theory, the dynamics of the constrained variables should be worked

\footnotetext{
${ }^{5}$ Note that imposing the condition of unitarity on the physical states, restricts $n$ to positive integer values, which in this case guarantees that the energy states are bounded from bellow. Therefore, besides the quantum algebra of observables, some other physical requirements such as unitarity principle play important roles in determining the set of physical states.

${ }^{6}$ If the constrained quantities are assumed as vanishing operators in the quantization procedure, then their brackets with the Hamiltonian should also vanish. On the other hand, if one quantizes the theory by imposing the condition that quantum operators corresponding to constraints should kill the physical states, then again it is clear that their brackets with the Hamiltonian should also kill the physical states.
} 
out completely before quantization, so that the final brackets of the Hamiltonian with the constraints vanish. This means that using the classical equations of motion, all the secondary constraints should be computed before quantization. Schematically we can say

primary constraints + classical equations of motion $\longrightarrow$ secondary constraints.

This point can be seen clearly in the example of the bosonic string with Neumann boundary conditions. If one had considered just the primary constraints $\left.\partial_{\sigma} X(\sigma, \tau)\right|_{\sigma=0, l}=0$ instead of the whole set of (5.7), then one would not have been able to deduce the expansion (5.12) for the fields. However, if one considers the primary constraints together with the equations of motion (resulting from the Hamiltonian (2.8) with $B=0$ ) one obtains

$$
\begin{aligned}
& \partial_{\tau} X(\sigma, \tau)=\Pi(\sigma, \tau) \\
& \partial_{\tau} \Pi(\sigma, \tau)=\partial_{\sigma}^{2} X(\sigma, \tau) .
\end{aligned}
$$

Then one can easily check that

$$
\begin{aligned}
& \partial_{\tau}\left(\partial_{\sigma} X(\sigma, \tau)\right)=\partial_{\sigma}\left(\partial_{\tau} X(\sigma, \tau)\right)=\partial_{\sigma} \Pi(\sigma, \tau) \\
& \partial_{\tau}\left(\partial_{\sigma} \Pi(\sigma, \tau)\right)=\partial_{\sigma}\left(\partial_{\tau} \Pi(\sigma, \tau)\right)=\partial_{\sigma}^{3} X(\sigma, \tau) .
\end{aligned}
$$

In this way the infinite set of constraints(5.7) are in fact resulting from the primary constraints plus the equations of motion (see (6.1)). This argument shows that there is no way to escape the fact that an infinite number of constraints really exist. If one uses the full capacity of the classical equations of motion (6.2), or the explicit form of the Hamiltonian in terms of the normal coordinates as

$$
H=\frac{1}{2} \sum_{n=0} \widetilde{c}_{n} c_{n}+\frac{1}{2} \sum_{n=1} \widetilde{a}_{n} a_{n}\left(\frac{n \pi}{l}\right)^{2},
$$

in order to determine the dynamics of the physical variables $a_{n}(\tau)$ and $c_{n}(\tau)$, then one obtains $\dot{a}_{n}=c_{n}$ and $\dot{c}_{n}=-(n \pi / l)^{2} a_{n}$, which acquire the solution

$$
\begin{aligned}
& a_{n}(\tau)=a_{n}(0) \cos \left(\frac{n \pi}{l} \tau\right)+\frac{l}{n \pi} c_{n}(0) \sin \left(\frac{n \pi}{l} \tau\right) \\
& c_{n}(\tau)=c_{n}(0) \cos \left(\frac{n \pi}{l} \tau\right)-\frac{n \pi}{l} a_{n}(0) \sin \left(\frac{n \pi}{l} \tau\right) .
\end{aligned}
$$

However, we insist again that the full dynamics of the physical variables is not necessary to quantize the theory. It seems that this partial role of the classical equations of motion in determining the dynamics of the constraints (or boundary conditions in most of the familiar physical systems) is the hidden reason behind the common practice of expanding the fields in terms of the classical solutions of equations of motion before quantization.

Now let us consider again the string in background $B$-field to observe the above points. In this case the equations of motion resulting from the Hamiltonian(2.8) read

$$
\begin{aligned}
& \partial_{\tau} X(\sigma, \tau)=\Pi(\sigma, \tau)-B \partial_{\sigma} X(\sigma, \tau) \\
& \partial_{\tau} \Pi(\sigma, \tau)=B \partial_{\sigma} \Pi(\sigma, \tau)+M \partial_{\sigma}^{2} X(\sigma, \tau)
\end{aligned}
$$


This gives

$$
\begin{gathered}
\partial_{\tau}(\Phi(\sigma, \tau))=\partial_{\sigma}(\Pi(\sigma, \tau)) \\
\partial_{\tau}\left(\partial_{\sigma} \Pi(\sigma, \tau)\right)=\partial_{\sigma}^{2}(\Phi(\sigma, \tau))
\end{gathered}
$$

In this way the full set of infinite constraints (5.15) emerge as the result of combining the primary constraints $\left.\Phi(\sigma, \tau)\right|_{\sigma=0, l}=0$ with the equations of motion (6.6). Using the expansion (5.20), the canonical Hamiltonian (2.8) in terms of the normal coordinates can be calculated as

$$
H=\frac{1}{2} \sum_{n=0}^{\infty} \widetilde{c}_{n} M^{-1} c_{n}+\frac{1}{2} \sum_{n=1}^{\infty} \widetilde{a}_{n} M a_{n}\left(\frac{n \pi}{l}\right)^{2} .
$$

We observe again that the full content of the dynamics is not needed for the quantization process. In fact, using the Hamiltonian (6.8), or the equations of motion (6.6), to determine the dynamics of the physical variables $a_{n}(\tau)$ and $c_{n}(\tau)$, one finds

$$
\begin{aligned}
& \dot{a}_{n}(\tau)=M^{-1} c_{n}(\tau) \\
& \dot{c}_{n}(\tau)=-\left(\frac{n \pi}{l}\right)^{2} M a_{n}(\tau) \quad n=0,1, \cdots
\end{aligned}
$$

which acquire the solution

$$
\begin{aligned}
& a_{n}(\tau)=a_{n}(0) \cos \left(\frac{n \pi}{l} \tau\right)+\frac{l}{n \pi} M^{-1} c_{n}(0) \sin \left(\frac{n \pi}{l} \tau\right) \\
& c_{n}(\tau)=c_{n}(0) \cos \left(\frac{n \pi}{l} \tau\right)-\frac{n \pi}{l} M a_{n}(0) \sin \left(\frac{n \pi}{l} \tau\right)
\end{aligned}
$$

and

$$
\begin{aligned}
& a_{0}(\tau)=M^{-1} c_{0}(0) \tau+a_{0}(0) \\
& c_{0}(\tau)=c_{0}(0) .
\end{aligned}
$$

As is apparent in order to quantize the theory, specially finding the important results of (5.23), one does not need to know the explicit time dependence given in Eq. (6.10). Also note that $c_{0}$ is constant, in agreement with our previous trick of adding the constant term $M^{-1} B c_{0} l / 2$ to the expansion of coordinate field in Eq. (5.20).

\section{Conclusion}

In this paper we discussed different aspects of the idea of considering the boundary conditions as Dirac constraints. Our theoretical laboratory for this aim was " an open string in a background $B$-field". We observed that besides the singularity of the Lagrangian, the boundary conditions can serve as a source of introducing the primary constraints. Analyzing in detail the discretized version of the model shows that the primary constraints are the continuum limit of the equations of motion for the end points; while the secondary constraints are derived by imposing the consistency conditions on these equations and 
then going to the continuum limit. In this process the continuity hypotheses is important. This implies that in order to get a continuum solution, the fields in the adjacent points in the corresponding discrete model should not differ drastically. In this way it turns out that the discretized model highly supports the existence of infinite chains of second class constraints.

The continuity hypotheses is also deeply related to Fourier expansion, as follows. It is well-known that in writing any field as a summation over the set of well-behaving continuous sine and cosine functions, any finite discontinuity in the field or in a finite number of its derivatives, is not seen by the expansion and is somehow removed from the problem. However, the boundary conditions should not be considered as such discontinuities. Although boundary conditions imposes some restrictions just on definite points at the border of the medium (i.e. end points of the string), they have their considerable influence throughout the whole medium. The important point is that the Fourier expansion plays the role of a carrier of boundary conditions from the boundaries through the medium. In fact, the emergence of an infinite number of constraints causes serious restrictions on the Fourier modes invited to the expansion of the fields. Since the Fourier modes are alive in the whole medium as continuous and well-behaved function, the message of boundary conditions is distributed in this way throughout the system. The familiar example in this regard is an open string with Neumann boundary conditions, in which the fields are expanded in terms of a set of discrete cosine modes only. In this case the Fourier expansion is, in fact, used to soften and flatten the fields undergoing definite conditions at the boundaries.

Summarizing, the continuity hypotheses implies, in the discrete model, the validity of constraints should be spread in a set of countable infinite number of adjacent points near the boundaries. This fact then shows itself in emerging infinite number of constraints; and finally causes omitting a large class of Fourier modes, which leads somehow to propagation of the effect of boundary conditions through the medium.

Another aim of this paper was studying the Poisson structure of the reduced phase space. Using the original coordinates of phase space implies serious difficulties in computing the complicated Dirac brackets. We observed that Fourier modes can serve as the normal coordinates of the reduced phase space. Using the Fourier modes make it possible to do calculations. Moreover, it gives a valuable understanding of the Poisson structure of the reduced phase space which contains the true physical degrees of freedom of the model. In this way after disappearing some Fourier modes as redundant (constrained) coordinates, the remaining modes can be viewed as the physical degrees of freedom, i.e. the coordinates of the reduced phase space. Fortunately these modes emerge as conjugate pairs with a simple and well-established bracket. As explained in the text, the brackets of the remaining modes define the Dirac brackets of fields. In this way one expands all associated fields and quantities just in terms of the normal coordinates, and then using their brackets one writes down all the Dirac brackets. It turns out that for an open string with mixed boundary conditions there remains no doubt about the fact that the Dirac 
brackets of the coordinate fields at the boundaries of the string are nonzero due to the $B$-field. Then, upon quantization, the coordinates of the string, and hence the coordinates of the D-brane, are non-commutative.

Another new feature in our approach is that we do not use of solutions of equations of motion in the process of quantization. We showed clearly, in the model under consideration, that it is in fact possible to find the algebraic structure of the quantum theory without any need to expand the fields in terms of solutions of classical equations of motion. We argued that in any quantum theory, constructed upon quantization of a classical model, one only needs to consider the dynamics of the constraints before quantization. In other words, it is not essential to find the dynamics of the complete set of physical variables to do quantization.

We think that our approach may be useful in analyzing the physical structure together with quantization of every model with complicated boundary conditions. Two recent examples can be seen in $[25,26]$. This approach may be applied also to more complicated systems such as membranes [27, 28, 29].

\section{Acknowledgements}

The authors would like to thank M. M. Sheikh-Jabbari for his useful discussions and comments and A. E. Mosaffa for reading the manuscript.

\section{References}

[1] P. A. M. Dirac, "Lecture Notes on Quantum Mechanics", Yeshiva University New York, 1964. Also see, P. A. M. Dirac, Proc. Roy. Soc. London, ser. A, 246, 326 (1950).

[2] M. Henneaux and C. Teitelboim, Quantization of Gauge Systems Princton Univ. Press, 1992.

[3] F. Ardalan, H. Arfaei, M. M. Sheikh-Jabbari, J. High Energy Phys. 02 (1999) 016, hep-th/9810072.

[4] F. Ardalan, H. Arfaei, M. M. Sheikh-Jabbari, Nucl. Phys. B 576 (2000) 578-596, hep-th/9906161.

[5] C.-S. Chu, P.-M. Ho, Nucl. Phys. B 568 (2000) 447-456, hep-th/9906192.

[6] N. Seiberg, E. Witten, J. High Energy Phys. 09 (1999) 032, hep-th/9908142.

[7] V. Schomerus, J. High Energy Phys. 06 (1999) 030, hep-th/9903205.

[8] A. Abouelsaood, C. G. Callan, C. R. Nappi and S. A. Yost, Nucl. Phys. B 280 (1987) 599-624.

[9] M.M. Sheikh-Jabbari, A. Shirzad, Eur. Phys. J. C 19 (2001) 383-390, hepth/9907055.

[10] W. T. Kim, J. J. Oh, Mod. Phys. Lett. A 15 (2000) 1597-1604, hep-th/9911085.

[11] N. R. F. Braga, C. F. L. Godinho, Phys. Rev. D 65 (2002) 085030, hep-th/0110297. 
[12] T. Lee, Phys. Rev. D 62 (2000) 024022, hep-th/9911140.

[13] T. Lee, Phys. Lett. B 483 (2000) 277-283, hep-th/0004159.

[14] A. Kokado, G. Konisi, T. Saito, Prog. Theor. Phys. 104 (2000) 1289-1308, hepth/0009190.

[15] R. Banerjee, B. Chakraborty, S. Ghosh, Phys. Lett. B 537 (2002) 340-350, hepth/0203199.

[16] W. He, L. Zhao, Phys. Lett. B 570 (2003) 251-259, hep-th/0307002.

[17] F. Loran, A. Shirzad, Int. J. Mod. Phys. A 17 (2002) 625-642, hep-th/0003010.

[18] Z. Yin, Phys. Lett. B 466 (1999) 234-238, hep-th/9908152.

[19] F. Loran, Phys. Lett. B 544 (2002) 199-201, hep-th/0207025.

[20] K. I. Tezuka, Eur. Phys. J. C 25 (2002) 465-468, hep-th/0201171.

[21] J. Jing, Z.-W. Long, L.-J. Tian, S. Jin, Eur. Phys. J. C 29 (2003) 447-451.

[22] C.-S. Chu, P.-M. Ho, Nucl. Phys. B 550 (1999) 151-168, hep-th/9812219.

[23] C.-S. Chu, F. Zamora, J. High Energy Phys. 02 (2000) 022, hep-th/9912153.

[24] L. Faddeev and R. Jackiw, Phys. Rev. Lett. 60, (1988) 1692.

[25] J. Jing, Eur. Phys. J. C 39 (2005) 123-127.

[26] J. Jing, Phys. Rev. D 71 (2005) 025023.

[27] S. Kawamoto, N. Sasakura, J. High Energy Phys. 07 (2000) 014, hep-th/0005123.

[28] A. Das, A. Melikyan, J. Maharana, J. High Energy Phys. 04 (2001) 016, hepth/0103229.

[29] R. Banerjee, B. Chakraborty, K. Kumar, Nucl. Phys. B 668 (2003) 179-206, hepth/0306122. 